\title{
Searches for rare charm decays at $\mathrm{LHCb}$
}

\section{Dominik Stefan Mitzel ${ }^{* \dagger}$}

Ruprecht-Karls-Universität Heidelberg (DE)

E-mail: dominik.mitzelecern.de

Rare and forbidden charm decays are unique probes for hints of physics beyond the Standard Model. The LHCb collaboration has made significant contributions to the field over the last years. This report presents results of the search for the lepton-flavour violating decay $D^{0} \rightarrow e^{ \pm} \mu^{\mp}$ and the first observations of the rare four body decays $D^{0} \rightarrow K^{-} \pi^{+} \mu^{+} \mu^{-}, D^{0} \rightarrow \pi^{+} \pi^{-} \mu^{+} \mu^{-}$and $D^{0} \rightarrow K^{+} K^{-} \mu^{+} \mu^{-}$.

EPS-HEP 2017, European Physical Society conference on High Energy Physics 5-12 July 2017

Venice, Italy

* Speaker.

${ }^{\dagger}$ on behalf of the LHCb Collaboration 


\section{Introduction}

The field of rare charm decays covers a wide variety of processes, ranging from lepton flavour (LFV) and lepton number violating (LNV) decays, which are strictly forbidden in the Standard Model (SM), to radiative decays which have branching fractions (BFs) in the order of $10^{-4}$. LFV and LNV decays can only be observed if contributions from processes not explainable in the frame of the SM are present, so that any signal would be a clear indication of new physics.

Another place to scrutinize the validity of the SM is the study of decays involving contributions of flavour-changing neutral currents (FCNC), which in the SM can only happen at loop level. Thus, they are sensitive to potentially additional and yet unobserved heavy particles, which might currently not be accessible in direct searches. Due to the highly effective Glashow-Illiopoulus-Maiani (GIM) mechanism [1], the supression of these kind of decays in the charm system is significantly higher than in the beauty and kaon sectors. However, the neutral charm system offers one of the few possibilities to test FCNCs acting on up-type quarks, which generically can be subject to different couplings than the down-type quarks, and therefore constitutes a complementary approach.

In the SM, the amplitudes of four body decays of the form $D^{0} \rightarrow h^{+} h^{-} \mu^{+} \mu^{-}(h=K, \pi)$ receive FCNC contributions which correspond to $\mathrm{BF}<\mathscr{O}\left(10^{-9}\right)$ [2], while the total $\mathrm{BF}$ is expected to be dominated by tree level amplitudes. In the tree level transition, the two muons come from the decay of an intermediate resonance and the BFs can reach up to $\mathscr{O}\left(10^{-6}\right)[3,2,4]$, depending on the specific final state.

Thanks to the huge charm production cross section at the Large Hadron Collider and the outstanding performance of the $\mathrm{LHCb}$ detector [5, 6], comprising excellent tracking, vertexing and particle identification, the LHCb collaboration has became a key player in charm physics and has recently made oustanding contributions to the field of rare charm decays. Only a small selection of analyses performed by $\mathrm{LHCb}$ is presented in this note.

First, the search for the LFV decay $D^{0} \rightarrow e^{ \pm} \mu^{\mp}$ [7] is presented in section 2. For this decay, some SM extensions even predict rates in the range $10^{-14}-10^{-6}$ [8]. Second, section 2 show the results of the first observation of the decay $D^{0} \rightarrow K^{-} \pi^{+} \mu^{-} \mu^{+}$in the $\rho^{0}-\omega$ region of the dimuon mass spectrum. This decay mode was used as a normalization for the BF measurement in the analysis of $D^{0}$ meson decays to $K^{+} K^{-} \mu^{+} \mu^{-}$and $\pi^{+} \pi^{-} \mu^{+} \mu^{-}$final states, which corresponds to the first observation of these decay modes. Details are given in section 4.

\section{Search for the lepton-flavour violating decay $D^{0} \rightarrow e^{ \pm} \mu^{\mp}$}

To search for the lepton-flavour violating decay $D^{0} \rightarrow e^{ \pm} \mu^{\mp}$ LHCb has analyzed its full Run 1 data set, corresponding to $1 \mathrm{fb}^{-1}$ collected in 2011 and $2 \mathrm{fb}^{-1}$ in 2012 at center of mass energies of $7 \mathrm{TeV}$ and $8 \mathrm{TeV}$, respectively. For this analysis, LHCb only selected $D^{0}$ mesons arising from the decay chain $D^{*+} \rightarrow D^{0} \pi^{+}$and performed the measurement relative to the normalization decay $D^{0} \rightarrow K^{-} \pi^{+}$, which has a similar topology and a well-measured BF [9]. In the ratio of BFs the production cross section as well as many detector induced effects cancel to a large extent and therefore help to reduce the systematic uncertainties. Background candidates from hadronic decays of the form $D^{0} \rightarrow \pi^{-} \pi^{+}$, with the pions misidentified as an electron-muon pair, are supressed by tight criteria on particle identification. The signal yields are determined using extended maximum- 
likelihood fits to the unbinned distributions of the resonstructed $D^{0}$ meson mass, $m\left(D^{0}\right)$, and the mass difference of the $D^{*+}$ and the $D^{0}$, hereafter denoted as $\Delta m$. Separation of background from randomly associated unrelated particles and signal is optimized by means of a multivariate analysis, which is used to divide the data sample in three subsamples. The ranges are chosen such that the separation between the background-only and signal-plus-background hypotheses is maximal. No significant signal was observed in the overall mass spectrum nor in any of the subsamples and an upper limit using the $C L_{s}$ method [10] was set. Figure 1 shows the observed $C L_{s}$ as well as the expected $C L_{s}$ with uncertainty bands as a function of the signal BF. All systematic uncertainties are propagated to the final limit as Gaussian constraints on the appropriate parameters. The final limit is determined to be

$$
\mathscr{B}\left(D^{0} \rightarrow e^{ \pm} \mu^{\mp}\right)<1.3 \times 10^{-8} \text { at } 90 \% \text { C.L. }
$$

This is the most stringent limit to date and corresponds to an improvement of one order of magnitude with respect to the previous results by Belle [11].

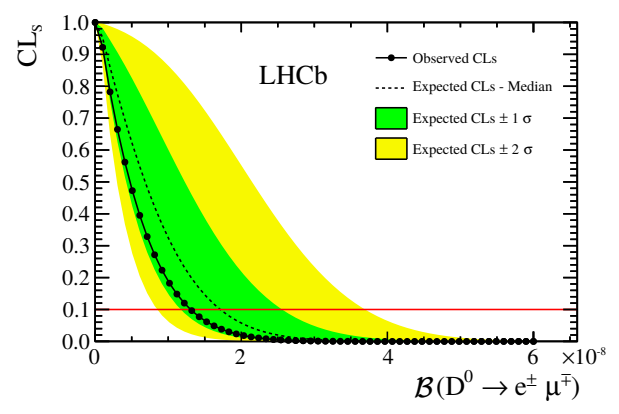

Figure 1: Observed (solid curve) and expected (dashed curve) $\mathrm{CL}_{s}$ values as a function of the signal $\mathrm{BF}$. The upper limit at the $90 \% \mathrm{CL}$ is indicated by the red line.

\section{First observation of the decay $D^{0} \rightarrow K^{-} \pi^{+} \mu^{-} \mu^{+}$in the $\rho^{0}-\omega$ region of the dimuon mass spectrum}

The BF measurement of the decay $D^{0} \rightarrow K^{-} \pi^{+} \mu^{-} \mu^{+}$by LHCb [12] represents the first observation of a four body-decay of a neutral charm meson having an oppositely charged muon pair in the final state. Since the decay amplitude in the $\rho^{0}$ - $\omega$ region of the dimuon mass spectrum, defined as $675 \mathrm{MeV} / c^{2}<m\left(\mu^{+} \mu^{-}\right)<875 \mathrm{MeV} / c^{2}$, is totally dominated by tree level transitions, the sensitivity to discover contributions from Standard Model extensions is somewhat limited. However, due to its decay topology, the decay channel serves as an ideal normalization mode for further $D^{0} \rightarrow h^{-} h^{+} \mu^{-} \mu^{+}(h=K, \pi)$ BF measurements. LHCb has analyzed collision data corresponding to $2 \mathrm{fb}^{-1}$ taken in 2012 and has selected $D^{0}$ mesons directly produced in the primary proton-proton interaction. The BF measurement was performed using the decay $D^{0} \rightarrow K^{-} \pi^{+} \pi^{-} \pi^{+}$as normalization, which has a BF [13] approximately four orders of magnitude higher than the expected signal BF. Decays of $D^{0} \rightarrow K^{-} \pi^{+} \pi^{-} \pi^{+}$can also enter the signal sample if two oppositely charged pions are misidentified as muons and pose one of the main background sources. Therefore, tight particle identification criteria are optimised simultaniously with a multivariate selection, designed to supress non-peaking background from combinations of unrelated charged particles. The yields of the 
signal and normalization mode are determined with a simultaneous maximum-likelihood fit to the binned $m\left(D^{0}\right)$ distributions of both decays. Figure 2 shows the $m\left(D^{0}\right)$ spectra with fit projection overlaid for the normalization (left) and signal (right) decays. The BF is measured to be

$$
\mathscr{B}\left(D^{0} \rightarrow K^{-} \pi^{+} \mu^{-} \mu^{+}\right)=(4.12 \pm 0.12 \pm 0.38) \times 10^{-6}
$$

where the first uncertainty is statistic and the second systematic. The result is in agreement with theoretical prediction [4].
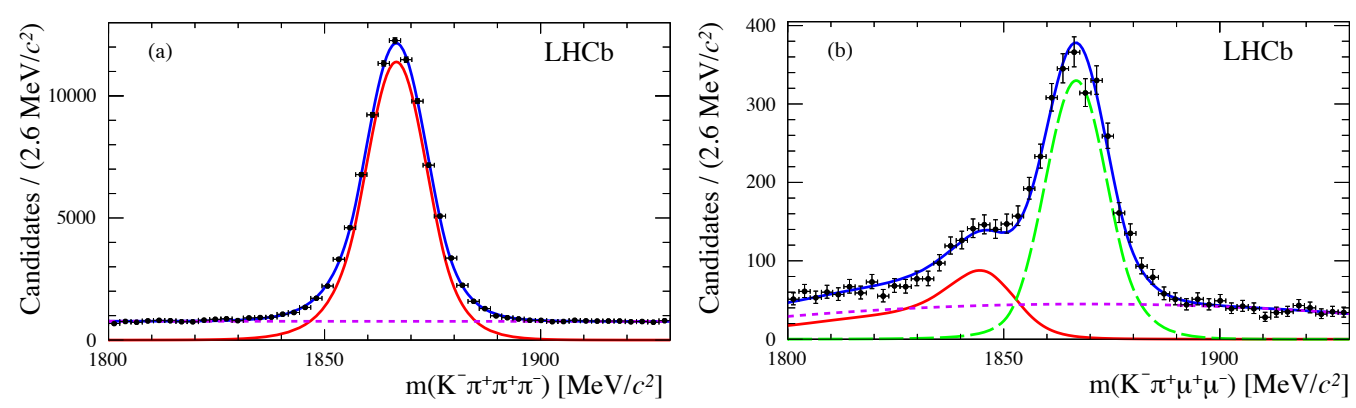

Figure 2: Distributions of $m\left(D^{0}\right)$ for $D^{0} \rightarrow K^{-} \pi^{+} \pi^{-} \pi^{+}$(left) and $D^{0} \rightarrow K^{-} \pi^{+} \mu^{+} \mu^{-}$(right) candidates with fit projection overlaid.

\section{Observation of $D^{0}$ meson decays to $K^{+} K^{-} \mu^{+} \mu^{-}$and $\pi^{+} \pi^{-} \mu^{+} \mu^{-}$final states}

For the first time at the EPS-HEP 2017, LHCb has presented the first observation of the decays $D^{0} \rightarrow \pi^{+} \pi^{-} \mu^{+} \mu^{-}$and $D^{0} \rightarrow K^{+} K^{-} \mu^{+} \mu^{-}$[14] using a sample of proton-proton collisions recorded in 2012 corresponding to $2 \mathrm{fb}^{-1}$. The measurement is performed in bins of dimuon mass to gain sensitivity to the FCNC processes contributing to the full decays amplitude. However, the long tails of the intermediate resonances span over the entire dimuon mass range. According to the known resonances, 5(3) bins are defined for the decay $D^{0} \rightarrow \pi^{+} \pi^{-} \mu^{+} \mu^{-}\left(D^{0} \rightarrow K^{+} K^{-} \mu^{+} \mu^{-}\right)$. These regions are: (low mass) $<525 \mathrm{MeV} / c^{2},(\eta) 525 \mathrm{MeV} / c^{2}-565 \mathrm{MeV} / c^{2},\left(\rho^{0} / \omega\right) 565 \mathrm{MeV} / c^{2}-$ $950 \mathrm{MeV} / c^{2},(\phi) 950 \mathrm{MeV} / c^{2}-1100 \mathrm{MeV} / c^{2}$, (high mass) $>1100 \mathrm{MeV} / c^{2}$, where the restricted phase space limits the $D^{0} \rightarrow K^{+} K^{-} \mu^{+} \mu^{-}$analysis to the first three bins. The decay $D^{0} \rightarrow K^{-} \pi^{+} \mu^{-} \mu^{+}$ was used as normalization mode, using the LHCb result presented in section 3. As the $D^{0}$ mesons are required to arise from the decay chain $D^{*+} \rightarrow D^{0} \pi^{+}$, restricting to candidates with $\Delta m$ in the range $144.5-146.5 \mathrm{MeV} / c^{2}$ very efficiently supresses background from randomly associated particles. The muons are selected by particle identification critera to significantly reduce the amount of hadronic $D^{0} \rightarrow h^{+} h^{-} \pi^{+} \pi^{-}$decays, where two pions are misidentified as muons. Residual background from combinations of unrelated particles is further reduced by a multivariate analysis. The signal yields are obtained through maximum-likelihood fits to the unbinned $m\left(D^{0}\right)$ distributions in each dimuon-mass regions. Figure 3 shows the $m\left(D^{0}\right)$ distribution of both signal decays in the dimuon-mass ranges with fit projection overlaid. A BF is quoted if an excess of candidates with respect to the background-only hypothesis with a significance of more than three standard deviations is observed. This happens for all dimuon-mass regions except for the $\eta$ range of both signal decays 

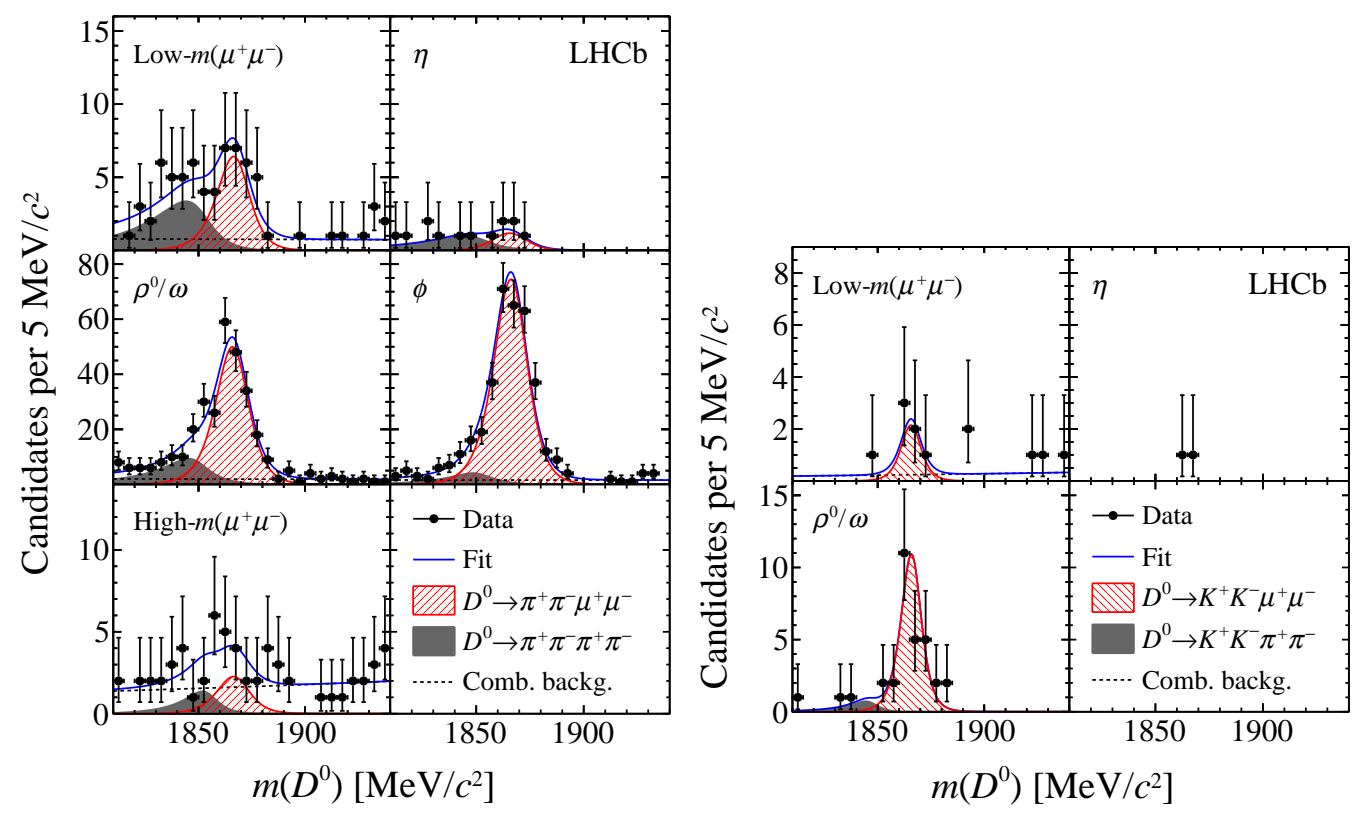

Figure 3: Distributions of $m\left(D^{0}\right)$ for the $D^{0} \rightarrow \pi^{-} \pi^{+} \mu^{-} \mu^{+}$(left) and $D^{0} \rightarrow K^{+} K^{-} \mu^{+} \mu^{-}$(right) candidates in the dimuon mass ranges. Fit projections are overlaid. No fit is performed in the $\eta$ region of $D^{0} \rightarrow$ $K^{+} K^{-} \mu^{+} \mu^{-}$, where only two candidates are observed.

and for the high mass region of $D^{0} \rightarrow \pi^{+} \pi^{-} \mu^{+} \mu^{-}$. In the regions with no significant excess of signal, upper limits are set on the BFs including all systematic uncertainties. Table 1 summarizes the measured partial BFs and computed upper limits for both signal decays in the dimuon mass ranges. Taking correlations into account, the total BFs integrated over dimuon mass are reported to be:

$$
\begin{aligned}
& \mathscr{B}\left(D^{0} \rightarrow \pi^{-} \pi^{+} \mu^{-} \mu^{+}\right)=(9.64 \pm 0.48 \pm 0.51 \pm 0.97) \times 10^{-7} \\
& \mathscr{B}\left(D^{0} \rightarrow K^{-} K^{+} \mu^{-} \mu^{+}\right)=(1.54 \pm 0.27 \pm 0.09 \pm 0.16) \times 10^{-7}
\end{aligned}
$$

where the first uncertainty is statistical, the second systematic and the third due to the limited knowledge of the normalization mode BF. The results are in agreement with theoretical predictions [4]. These are the rarest charm hadron decays measured to date.

\section{Summary}

The LHCb collaboration has a very succesful rare charm physics program and has made major contributions to the field analyzing the Run 1 data set collected in the years 2011 and 2012. Most of the recently published analyses report world's best measurements, of which only a subset is presented in this report. Analyses incorporating Run 2 data will follow soon and further improve limits on very rare and forbidden decays.

\section{References}

[1] S. L. Glashow, J. Iliopoulos and L. Maiani, Weak Interactions with Lepton-Hadron Symmetry, Phys. Rev. D2 (1970) 1285-1292. 


\begin{tabular}{lcc}
\hline & $D^{0} \rightarrow \pi^{+} \pi^{-} \mu^{+} \mu^{-}$ \\
$\mu^{+} \mu^{-}$region & {$\left[\mathrm{MeV} / \mathrm{c}^{2}\right]$} & $\mathscr{B}\left[10^{-8}\right]$ \\
Low mass & $<525$ & $7.8 \pm 1.9 \pm 0.5 \pm 0.8$ \\
$\eta$ & $525-565$ & $<2.4(2.8)$ \\
$\rho^{0} / \omega$ & $565-950$ & $40.6 \pm 3.3 \pm 2.1 \pm 4.1$ \\
$\phi$ & $950-1100$ & $45.4 \pm 2.9 \pm 2.5 \pm 4.5$ \\
High mass & $>1100$ & $<2.8(3.3)$ \\
\hline \multicolumn{3}{c}{$D^{0} \rightarrow K^{+} K^{-} \mu^{+} \mu^{-}$} \\
$\mu^{+} \mu^{-}$region & {$\left[\mathrm{MeV} / \mathrm{c}^{2}\right]$} & $\mathscr{B}\left[10^{-8}\right]$ \\
Low mass & $<525$ & $2.6 \pm 1.2 \pm 0.2 \pm 0.3$ \\
$\eta$ & $525-565$ & $<0.7(0.8)$ \\
$\rho^{0} / \omega$ & $>565$ & $12.0 \pm 2.3 \pm 0.7 \pm 1.2$ \\
\hline
\end{tabular}

Table 1: Branching fractions of (top) $D^{0} \rightarrow \pi^{+} \pi^{-} \mu^{+} \mu^{-}$and (bottom) $D^{0} \rightarrow K^{+} K^{-} \mu^{+} \mu^{-}$decays in different ranges of dimuon mass, where the uncertainties are statistical, systematic and due to the limited knowledge of the normalization branching fraction. The reported upper limits correspond to 90\% (95\%) CL.

[2] A. Paul, I. I. Bigi and S. Recksiegel, On $D \rightarrow X_{u} \ell^{+} \ell^{-}$within the standard model and frameworks like the littlest Higgs model with T parity, Phys.Rev. D83 (2011) 114006, [1101.6053].

[3] S. Fajfer, N. Košnik and S. Prelovšek, Updated constraints on new physics in rare charm decays, Phys.Rev. D76 (2007) 074010, [0 706 .1133].

[4] L. Cappiello, O. Cata and G. D'Ambrosio, Standard model prediction and new physics tests for $D^{0} \rightarrow h_{1}^{+} h_{2}^{-} \ell^{+} \ell^{-}(h=\pi, K ; \ell=e, \mu)$, JHEP 04 (2013) 135, [1209.4235].

[5] LHCв COLlaboration collaboration, A. A. Alves Jr. et al., The LHCb detector at the LHC, JINST 3 (2008) S08005.

[6] LHCb COllaboration collaboration, R. Aaij et al., LHCb detector performance, Int. J. Mod. Phys. A30 (2015) 1530022, [1412.6352].

[7] LHCb COllaboration collaboration, R. Aaij et al., Search for the lepton-flavour violating decay $\mathrm{D}^{0} \rightarrow \mathrm{e}^{ \pm} \mu^{\mp}$, Phys. Lett. B754 (2016) 167 LHCb-PAPER-2015-048, CERN-PH-EP-2015-306, [1512.00322].

[8] G. Burdman, E. Golowich, J. L. Hewett and S. Pakvasa, Rare charm decays in the standard model and beyond, Phys. Rev. D66 (2002) 014009, [hep-ph / 0112235].

[9] Particle Data Group collaboration, C. Patrignani et al., Review of Particle Physics, Chin. Phys. C40 (2016) 100001.

[10] A. L. Read, Presentation of search results: The CL(s) technique, J. Phys. G28 (2002) 2693-2704.

[11] Belle collaboration, M. Petric et al., Search for leptonic decays of $D^{0}$ mesons, Phys. Rev. D81 (2010) 091102, [1003.2345].

[12] LHCв COLLABORATION collaboration, R. Aaij et al., First observation of the decay $\mathrm{D}^{0} \rightarrow \mathrm{K}^{-} \pi^{+} \mu^{+} \mu^{-}$in the $\rho^{0}-\omega$ region of the dimuon mass spectrum, Phys. Lett. $\mathbf{B} 757$ (2016) 558 LHCb-PAPER-2015-043, CERN-PH-EP-2015-283, [1510 . 08367]. 
[13] CLEO COLlaboration collaboration, G. Bonvicini et al., Updated measurements of absolute $D^{+}$ and $D^{0}$ hadronic branching fractions and $\sigma\left(e^{+} e^{-} \rightarrow D \bar{D}\right)$ at $E_{\mathrm{cm}}=3774 \mathrm{MeV}$, Phys.Rev. D89 (2014) 072002, [1312.6775].

[14] LHCB collaboration, R. Aaij et al., Observation of $D^{0}$ meson decays to $\pi^{+} \pi^{-} \mu^{+} \mu^{-}$and $K^{+} K^{-} \mu^{+} \mu^{-}$final states, 1707.08377 . 